\title{
The developmental expression of the cholinergic-specific antigen Chol-1 in the central and peripheral nervous system of the rat
}

\author{
Edmund A. Derrington and Edilio Borroni \\ Arbeitsgruppe Neurochemie, Max-Planck-Institut für Biophysikalische Chemie, Göttingen (F.R.G.)
}

(Accepted 19 September 1989)

Key words: Hippocampus; Neuromuscular junction; Synaptogenesis; Cholinergic-specific antigen; Nerve terminal; Ganglioside

\begin{abstract}
Antisera raised by the injection into sheep of presynaptic plasma membranes isolated from the purely cholinergic electromotor nerve terminals of Torpedo marmorata recognize a cholinergic-specific epitope, designated Chol-1 which has been shown to be gangliosidic in nature both in Torpedo (Richardson et al., 1982) and guinea-pig brain (Ferretti and Borroni, 1986). In rat brain the serum recognizes a group of antigenically-related minor gangliosides (Chol- $1 \alpha, \beta$ and $\gamma$ ) which migrate just below the standard gangliosides GQ, GT1B and between GD1b and GD1a, respectively. We have studied the developmental expression of these gangliosides in rat brain and hippocampus and in the neuromuscular junction of rat intercostal muscle in an attempt to correlate their expression with specific events in the development of the cholinergic neuron. The period in which Chol-1 is first detected suggests that it is expressed relatively late during the maturation process of the cholinergic synapse. This is supported by the finding: (a) that it is not detected in the growth cones (immature nerve terminals) in 5-day-old rat brain but is in the whole brain implying that only the more mature nerve terminals present at this stage express Chol-1; and (b) that Chol-1 is first expressed in the neuromuscular junction at a time in which functional synapses are already present. These results argue against a role for the Chol-1 antigens as recognition molecules in the formation of cholinergic synapses. The expression of Chol-1 in both the hippocampus and the neuromuscular junction correlates well with the establishment of the adult pattern of innervation; thus the Chol-1 antigens may be seen as markers for mature cholinergic terminals.
\end{abstract}

\section{INTRODUCTION}

There is increasing evidence that carbohydrate antigens may serve as specific markers for different classes of neural cells. Thus galactocerebroside may be used as a marker for oligodendrocytes and Schwann cells ${ }^{21,33}$ and tetanus toxin which binds primarily to the gangliosides GD1b and GT1b, may be used as a neuron-specific probe $^{33}$. Furthermore, the expression of carbohydrate antigens has been observed to vary within the same cell type so that subpopulations of such cell types can be identified. Thus Dodd and Jessel ${ }^{9}$ have shown that complex glycolipids of the globo- and lacto-series specify subsets of neurons in the rat dorsal root ganglion and that the expression of these antigens on specific dorsal root ganglion cells correlates with their functional classification.

Previous work in our laboratory has led to the identification of a group of antigenically related minor gangliosides, collectively designated Chol-1, that appear to be exclusively localized on cholinergic neurons in the central and peripheral nervous systems ${ }^{2,12,31}$. These antigens were detected by the use of an antiserum
(anti-Chol-1 antiserum) raised against presynaptic plasma membranes derived from the purely cholinergic innervation of the electric organ of Torpedo marmorata ${ }^{22,37,47}$. This serum induced a selective complement-mediated lysis of the cholinergic subpopulation of mammalian brain synaptosomes indicating the expression of this antigen on the external surface of mammalian cholinergic nerve terminals ${ }^{2,35}$. Further support for the cholinergic specificity of this antigen came from the following findings: (a) anti-Chol-1 antiserum can be used to specifically stain cholinergic elements in the rat $\mathrm{CNS}^{31}$; (b) section of the fimbria, which causes a massive degeneration of cholinergic terminals in the hippocampus, leads to a concomitant fall in the level of Chol-1 in the hippocampus ${ }^{8}$; (c) the anti-Chol-1 antiserum can be used to affinity-purify the cholinergic subpopulation of synaptosomes from the total population of rat brain synaptosomes $^{36}$.

Recently, our interest has focused on the function of the Chol-1 gangliosides. Large changes in the concentration and distribution of gangliosides and other glycoconjugates have been observed during differentiation both in vitro and in vivo ${ }^{18,25}$, thus implicating them in diverse

Correspondence: E. Borroni, AG Neurochemie, Max-Planck-Institut für Biophysikalische Chemie, Postfach 2841, D-3400 Göttingen, F.R.G. 
processes in neural development such as cell adhesion ${ }^{27}$, neuritogenesis ${ }^{11,38}$ and synaptogenesis ${ }^{17.30}$. Most of these studies, however, have investigated changes in major gangliosides. The anti-Chol-1 antiserum provides the opportunity of studying minor gangliosidic antigens expressed only by cholinergic neurons. Their developmental expression may thus provide a marker for cholinergic neurons at a specific stage in their development. Furthermore, temporal correlation of the expression of Chol-1 with processes occurring during the maturation of the cholinergic system may well guide further investigation of their function.

We have accordingly quantified the developmental expression of the Chol-1 antigens in whole rat brain and hippocampus relative to the expression of the cholinergicspecific marker choline acetyl-transferase (ChAT), and present an immunohistochemical study of their expression in the neuromuscular junction ( $\mathrm{nmj}$ ) of intercostal muscle. Both the hippocampus ${ }^{29}$ and the neuromuscular junction ${ }^{7}$ provide systems in which the time course of the main events of cholinergic synaptogenesis have already been described.

\section{MATERIALS AND METHODS}

\section{Tissues and subcellular fractions}

Tissue preparation. Postnatal and adult Wistar albino rats were obtained from the Institute's breeding colony. Animals were killed by decapitation and dissected on ice. Material removed was immediately frozen in liquid nitrogen and stored at $-80^{\circ} \mathrm{C}$.

Preparation of growth cones. These were prepared by the method of Gordon-Weeks and Lockerbie ${ }^{16}$ from 5-day postnatal rat brain and were generously donated by Dr G.I. Kristjansson.

\section{Preparation and treatment of gangliosides}

Sources and chromatography. The polysialoganglioside fraction PSG 4 was obtained from Dr Pallmann GmbH (Munich, F.R.G.). Other gangliosides were prepared according to Tettamanti et al ${ }^{46}$ and their sialic acid content assayed by the resorcinol method ${ }^{43}$.

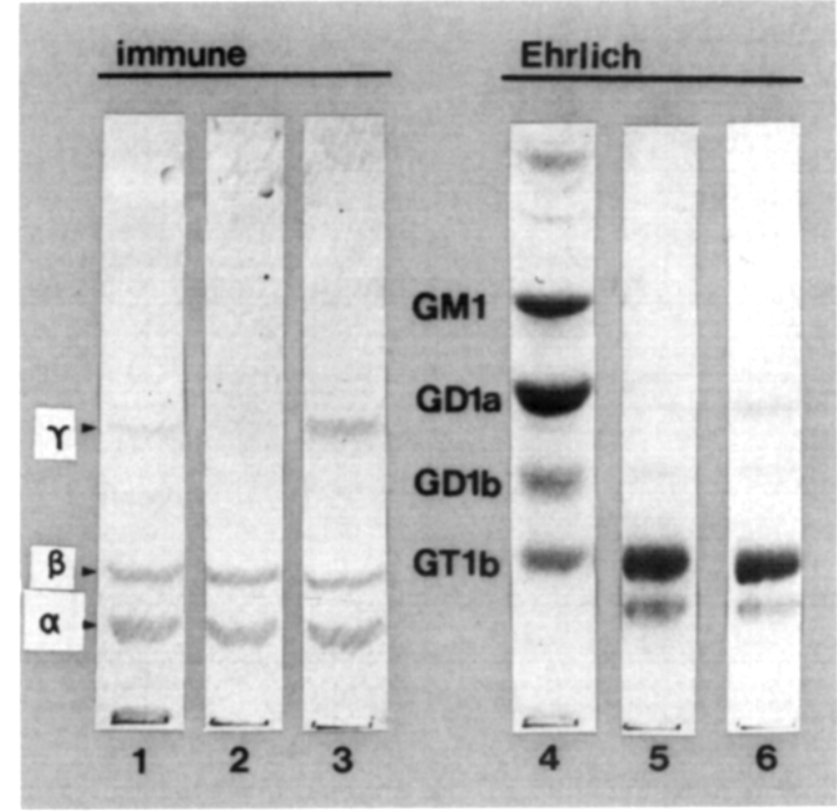

Fig. 1. The Chol-1 gangliosides in rat brain Adult rat brain gangliosides (lanes 1 and 4), PSG 4 gangliosides (lanes 2 and 5) and PSG 4 gangliosides which have been dialysed overnight against distilled water (lanes 3 and 6) are shown. Lanes 1, 2 and 3 show the result of immune staining with anti-Chol-1 antiserum and lanes 4,5 and 6 are lanes 1,2 and 3 rephotographed after staining with Ehrlich's reagent. Chol- $1 \alpha, \beta$ and $\gamma$ can be seen migrating below GQ, below GT1b and between GD1b and GDla respectively. Lane 3 shows that Chol- $1 \gamma$ can be formed by dialysis of a fraction which contains only Chol- $1 \alpha$ and $\beta$. The relative abundance of the major gangliosides in lane 4 is somewhat different from that reported in the literature. This is due to the fact that Ehrlich's reagent was applied after the completion of the immune staining. When chromatograms not previously submitted to immune staining were treated with Ehrlich's reagent a standard pattern was obtained (compare the pattern in lane 4 with that of lane 5 in Fig. 4).

Since further purification of the gangliosides by means of DEAE, Kieselgel and other chromatographic procedures do not alter the immunoreactivity of our antiserum toward the Chol- 1 bands we have not further purified the ganglioside fractions used in this study.

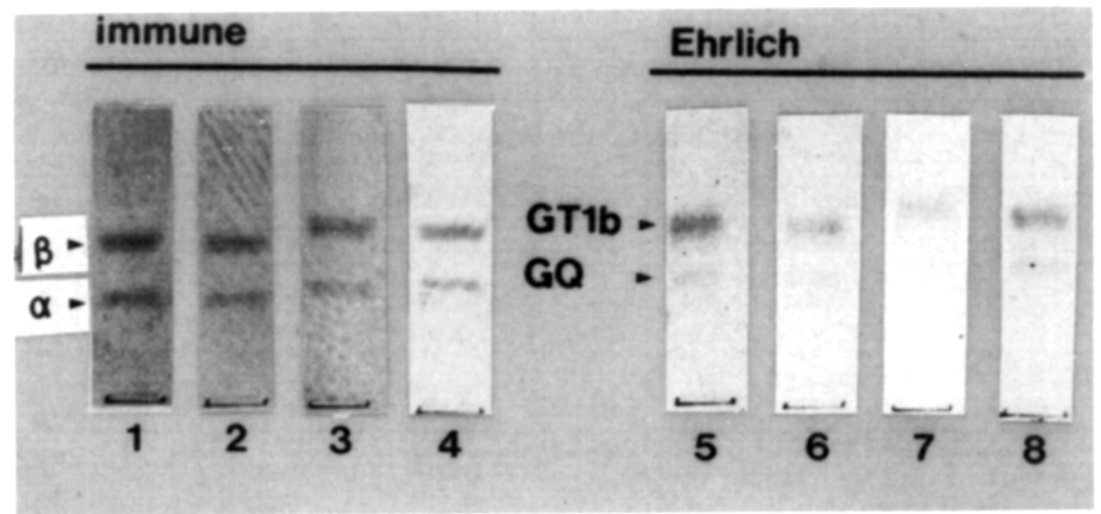

Fig. 2. Protease insensitivity of the Chol-1 gangliosides. The PSG 4 ganglioside fraction is shown separated on TLC following treatment with protease type VI (lanes 2 and 6), protease type V (lanes 3 and 7), trypsin (lanes 4 and 8 ) and control (lanes 1 and 5). Lanes 1-4 are the results of immune staining with anti-Chol-1 antiserum and lanes 5-8 are those of staining with Ehrlich's reagent using the same procedure as Fig. 1. Protease treatment has no apparent effect on the Chol-1 gangliosides since there is no significant change in immunoreactivity and in the chromatographic mobility of the gangliosides on the TLC. In all cases $\alpha$ and $\beta$ can be seen migrating just below GT1b and GQ. 
Ganglioside molecular species containing between 2 and $5 \mu \mathrm{g}$ sialic acid were separated by thin-layer chromatography (TLC) on Kieselgel 60 high performance TLC (HPTLC) plates (Merck, Darmstadt, F.R.G.) at $22^{\circ} \mathrm{C}$ using chloroform/methanol $/ 0.2 \%$ $\mathrm{CaCl}_{2}$ (40:42:11). Gangliosides were rendered visible, often after immune staining (see below), by spraying with p-dimethylaminobenzaldehyde (Ehrlich's reagent), followed by heating at $140^{\circ} \mathrm{C}$ for $15 \mathrm{~min}^{32}$. The plates were calibrated with bovine standard gangliosides (Seromed, Munich, F.R.G.). The nomenclature of gangliosides is that of Svennerholm ${ }^{44,45}$.

Neuraminidase digestion. Gangliosides extracted from rat brain were dissolved in $1 \mathrm{mM}$ acetate buffer, $\mathrm{pH} 5$, containing 1 unit of neuraminidase (type V: purified from Clostridium perfringens, Sigma, Munich, F.R.G.) per $50 \mu$ g of ganglioside-bound sialic acid and incubated at $37^{\circ} \mathrm{C}$ for $12 \mathrm{~h}$. A further 0.5 units of neuraminidase in the same buffer was added and the reaction mixture incubated for a further $6 \mathrm{~h}$ at $37^{\circ} \mathrm{C}$.

The mixture was then dialysed against $50 \mathrm{ml}$ of double-distilled water on a Millipore VSWP 013 filter (porosity $0.05 \mu \mathrm{m}$ ) for $5 \mathrm{~min}$, the residue was collected, lyophilized, resuspended in chloroform methanol $(2: 1 \mathrm{v} / \mathrm{v})$ and centrifuged in an Eppendorf table-top centrifuge to remove any debris. The solvent containing the gangliosides was stored at $-25^{\circ} \mathrm{C}$.

Protease treatment.Protease type V and type VI from Streptomyces griseus (Sigma, Munich, F.R.G.) were incubated with 200 $\mu \mathrm{g}$ PSG 4 at a concentration of 1 unit per $100 \mu \mathrm{g}$ ganglioside (dry weight) for $60 \mathrm{~min}$ at $37^{\circ} \mathrm{C}$ in Tris-buffered saline ( $\mathrm{pH}$ 7.9). Trypsin from bovine pancreas (Sigma, Munich, F.R.G.) was incubated with $200 \mu \mathrm{g}$ PSG $4(1 \mu \mathrm{g} / 50 \mu \mathrm{g})$ overnight at $37^{\circ} \mathrm{C}$ in Tris-buffered saline (pH 8.0). The above were then dialysed against $100 \mathrm{ml}$ of

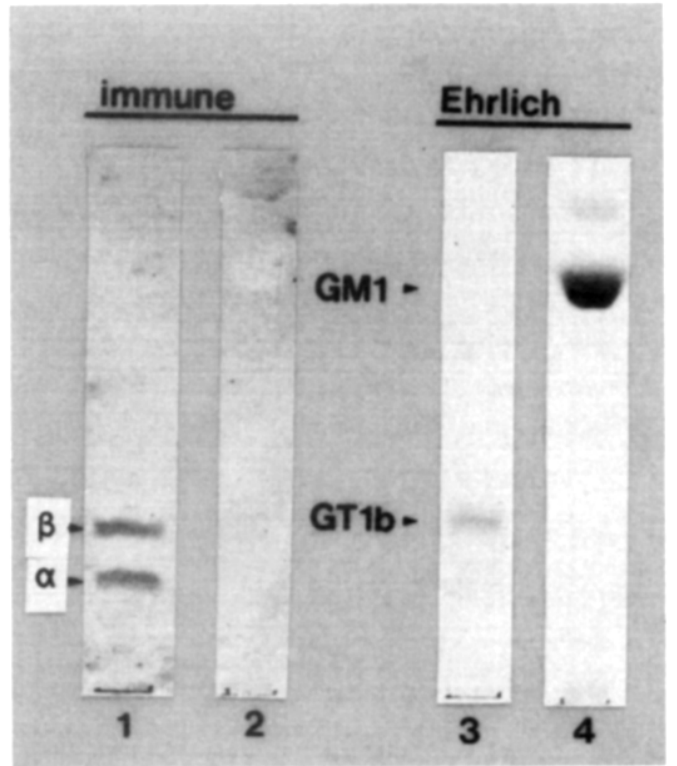

Fig. 3. Neuraminidase sensitivity of the Chol-1 gangliosides. PSG 4 gangliosides, with (lanes 2 and 4) and without (lanes 1 and 3) neuraminidase treatment are shown. Lanes 1 and 2 are immune stained with anti-Chol-1 antiserum and lanes 3 and 4 are lanes 1 and 2 after staining with Ehrlich's reagent. Neuraminidase treatment in the conditions described in 'Materials and Methods' will remove all sialic acid residues not bound directly to the oligosaccharide backbone of gangliosides; the effectiveness of this treatment can be seen by the change in migration of the Ehrlich-positive bands in PSG 4 from polysialogangliosides to GM1 and much more faintly above it GM2. Following such neuraminidase treatment immunoreactivity against Chol-1 is lost. This indicates the presence of sialic acid in Chol-1. Note also that a higher amount of gangliosides was loaded on lane 4 . double-distilled water on a Millipore VSWP 013 filter (porosity 0.05 $\mu \mathrm{m})$ for $5 \mathrm{~min}$, the residue collected, lyophilized, resuspended in chloroform/methanol $(2: 1 \mathrm{v} / \mathrm{v})$ and centrifuged in an Eppendorf table top centrifuge to remove any debris. The solvent containing the gangliosides was stored at $-25^{\circ} \mathrm{C}$.

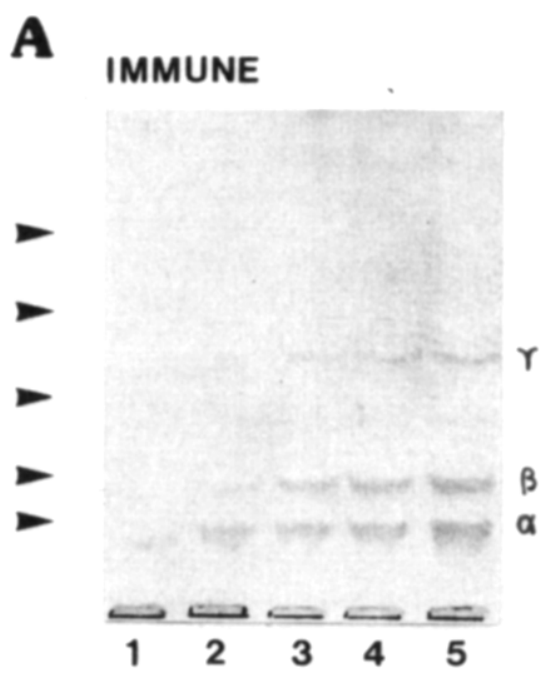

B

\section{EHRLICH}

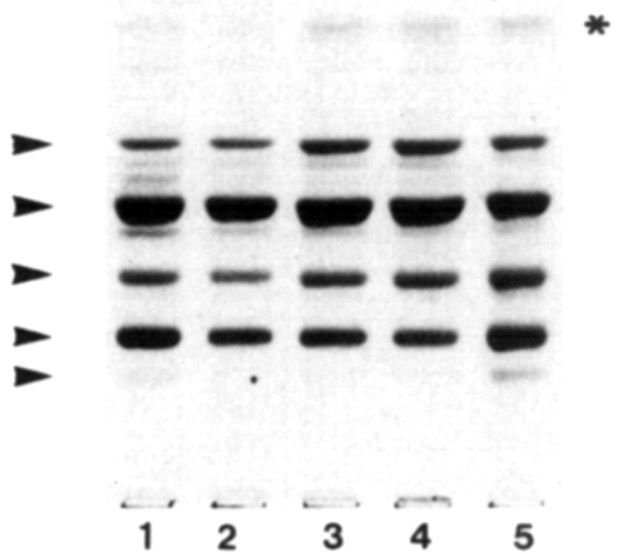

Fig. 4. The developmental expression of Chol-1 in rat brain. A: gangliosides prepared from $5,10,20$ and 30 day and adult brain containing 5, 5, 4, 3 and $3 \mu \mathrm{g}$ sialic acid, respectively were separated on TLC and immune stained with anti-Chol-1 antiserum (lanes 1-5). The expression of the immunoreactive Chol-1 gangliosides relative to the total ganglioside mixture can be seen to increase during development with Chol- $1 \alpha, \beta$ and $\gamma$ being first detected 5, 10 and 20 days postnatal, respectively. Different amounts of gangliosides were loaded in each lane so that the intensity of the immune staining on the TLC would be within the limits of the linearity of the system. Various concentrations of PSG 4 were run on the same TLC to check the linearity of the immune response and to enable the quantification of Chol-1 by densitometric scanning. B: gangliosides extracted from brains of the same developmental stages were separated on TLC ( $3 \mu \mathrm{g}$ of sialic acid per lane) and stained with Ehrlich's reagent (lanes 1-5, respectively). The band labelled by the asterisk is a contaminant of the preparation since it is stained yellow by Ehrlich's reagent. Arrowheads indicate the positions of standard gangliosides - from the top: GM1, GD1a, GD1b, GT1b and GQ. 
Immunochemical methods

Affinity purificaton of serum. Anti-Chol-1 antiserum was that described by Richardson et al.$^{37}$ and affinity purified as described by Ferretti and Borroni ${ }^{12}$. The affinity purification of the anti-Chol-1 antiserum is based on the observation that gangliosides extracted from the electric organ of Torpedo marmorata provide a richer source of antigen than mammalian brain gangliosides. Affinity purification of the antiserum on immobilized Torpedo electric organ gangliosides greatly increases its capacity to induce lysis of the cholinergic synaptosomes and renders it specific for the Chol-1 antigen $^{12}$. Briefly, Torpedo electric organ gangliosides containing 5 $\mathrm{mg}$ of ganglioside-bound sialic acid were covalently bound to long-chain alkylamine, controlled pore, glass beads (Pierce Chemicals, Rockford, U.S.A.) according to Young et al. ${ }^{48}$. The column was washed with $5 \mathrm{ml}$ of $10 \mathrm{mM}$ sodium phosphate buffer ( $\mathrm{pH} 7.4$ ) in $0.15 \mathrm{M} \mathrm{NaCl}$ (PBS), $3 \mathrm{ml}$ of KSCN and then $8 \mathrm{ml}$ of PBS; 100 $\mu \mathrm{l}$ of anti-Chol-1 antiserum were passed through the column 5 times and after extensive washing with PBS the bound antibodies were eluted with $1 \mathrm{ml}$ of $3 \mathrm{M} \mathrm{KSCN}$ and $2 \mathrm{ml}$ of PBS. The eluate was dialysed in the presence of BSA against PBS and stored in aliquots at $-20^{\circ} \mathrm{C}$.

Immune staining on $T L C$. Detection of antigenic gangliosides separated on aluminium-backed TLC (as described) was performed by a modification of the method of Brockaus et $\mathrm{al} .{ }^{4}$. A peroxidaselabelled rabbit anti-sheep Ig (Cappel Laboratories, Dinatech, Plochingen, F.R.G.) was used to detect the anti-Chol-1 antiserum bound to gangliosides. The reaction was developed by dipping the chromatogram in $0.01 \%$ 4-dichloro-1-naphthol and $0.9 \mathrm{mM} \mathrm{H}_{2} \mathrm{O}_{2}$ in PBS.

Quantification was performed using a Shimadzu C5-930 dual wave-length TLC scanner with a Shimadzu D2-2 data recorder. The limits of linearity of the immune response were established using the PSG 4 fraction which was found to be highly enriched with respect to Chol- $1^{8}$. Usually immune staining was followed by spraying the TLC with Ehrlich's reagent. Though the presence of the immunochemical reagents somewhat distorted the relative abundance of the various gangliosides this method was preferred in some experiments since it allowed a direct comparison of the chromatographic mobilities of the immunoreactive bands with those of the major gangliosides.
Immunohistochemistry. Unfixed cryostat sections of rat intercos tal muscle were fixed in acetone for $10 \mathrm{~min}$ at $-20{ }^{\circ} \mathrm{C}$ and after air drying were stained by conventional immunofluorescence techniques. Briefly, sections were incubated for $30 \mathrm{~min}$ in $1 \%$ BSA, $10 \%$ rabbit non-immune serum in PBS (PBSA), incubated $2 \mathrm{~h}$ in affinity-purified antibody diluted 3-fold in PBSA, washed 6 times for $5 \mathrm{~min}$ in PBS, then incubated $1 \mathrm{~h}$ with fluorescein isothiocyanateconjugated rabbit anti-sheep IgG diluted $1: 100$ in PBSA and $\alpha$-bungarotoxin (obtained from Boehringer, Mannheim, F.R.G.) diluted 1:20 in the same medium and labelled with tetramethyl rhodamine isothiocyanate according to the method of Ravdin and Axelrod ${ }^{34}$. Prior to use the second antibody was adsorbed against rat diaphragm membranes. The amount of $\mathrm{IgG}$ in the affinitypurified anti-Chol-1 antiserum was determined to be $30-50 \mu \mathrm{g} / \mathrm{ml}$, therefore an equivalent amount of $\mathrm{IgG}$ prepared from non-immune sheep serum was used as a control. A section treated with $\alpha$-bungarotoxin and non-immune sheep serum was included for each stage. This was done in order to control: (a) that the signal due to $\alpha$-bungarotoxin will not contribute to the Chol-1 immunoreactivity by passing through the fluorescein filter; and (b) the level of background staining due to non-specific binding.

Other assays. Whole rat brains and hippocampi were homogenized in PBS and diluted to a concentration of $1: 100(\mathrm{w} / \mathrm{v})$ in PBS. Samples $(50 \mu \mathrm{l})$ of this homogenate were assayed for ChAT according to Fonnum ${ }^{15}$. Their protein content was estimated according to Lowry ${ }^{26}$

\section{RESULTS}

\section{The Chol-1 antigen}

It has previously been shown that the cholinergicspecific antigenic determinant recognized by the antiChol-1 antiserum in guinea-pig brain is located on two gangliosides migrating on TLC just below GQ and $\mathrm{GT}^{1} \mathrm{~b}^{12}$. However, since the present study was carried out in rat, we felt it desirable to obtain direct evidence for
A

B

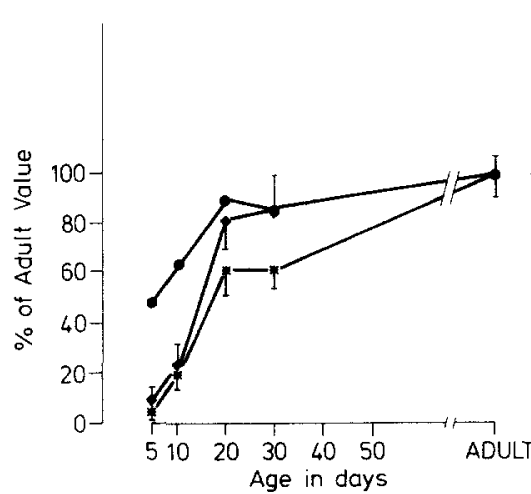

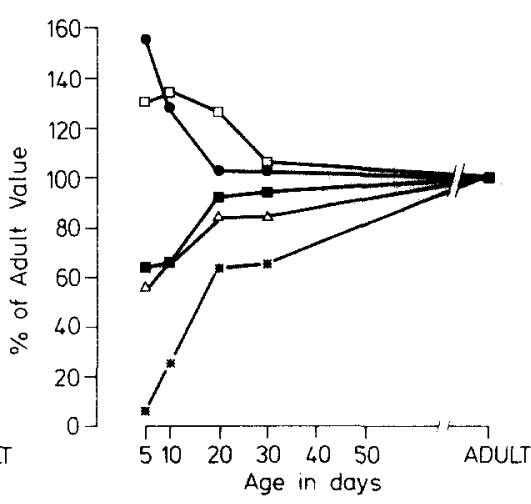

Fig. 5. A: the quantitative developmental expression of ChAT, Chol-1 and ganglioside-bound sialic acid in rat brain. Data are calculated per unit of wet weight and expressed as percentages of adult values. Circles, ganglioside-bound sialic acid; diamonds, ChAT; asterisks, Chol-1. For Chol-1 and ChAT, each point represents the mean \pm S.D. of 3 experiments; for sialic acid, the results of a typical experiment are shown. The expression of Chol-1 correlates very closely with that of ChAT during development though the latter reaches a plateau several days earlier. B: developmental expression of Chol-1 and major brain gangliosides. Equal amounts of ganglioside ( $5 \mu \mathrm{g}$ sialic acid) prepared from the various developmental stages were separated on TLC and stained with Ehrlich's reagent or immune-stained with anti-Chol-1 antiserum. The major brain gangliosides were quantified by densitometric scanning, calculated as molar concentrations and plotted as percentages of adult value. Chol-1 values (asterisks) were calculated as units per $5 \mu \mathrm{g}$ sialic acid and plotted as percentages of adult values. The developmental pattern of the major ganglioside is in agreement with that of previous reports ${ }^{28,42}$. Empty triangles, GM1; empty squares, GD1a; filled squares, GD1b; filled circles, GT1b. 
the presence of these antigenic components in this animal.

In rat brain the anti-Chol- 1 antiserum recognized three minor glycolipids, two migrating on TLC below GQ and GT1b (identical with those found in guinea pig) and a third, fainter component between GD1b and GD1a. These will be referred to as Chol- $1 \alpha, \beta$ and $\gamma$, respectively (Fig. 1). We strongly suspect, however, that Chol- $1 \gamma$ may be an artefactual component produced by the breakdown of either Chol- $1 \alpha$ or $\beta$ (or both). Fig. 1 shows gangliosides extracted from adult rat brain separated on TLC and stained with anti-Chol-1 antiserum followed by Ehrlich's reagent (lanes 1 and 4). A polysialoganglioside fraction (PSG 4) which we have found to be enriched with respect to the Chol- $1 \alpha$ and $\beta$ antigens is also shown (lanes 2 and 5). We have found that by simply dialysing the PSG 4 ganglioside fraction against distilled water overnight one can induce the formation of Chol- $1 \gamma$ (lanes 3 and 6). The reasons for this apparent sensitivity to dialysis are presently unclear. Experiments are in progress to clarify this point.

Amphiphilic glycoproteins and glycopeptides are known to copurify with gangliosides and migrate on TLC in a similar manner. In order to show that the Chol-1 antigens are not peptidic contaminants of our ganglioside preparations we have tested their sensitivity to protease digestion. Fig. 2 shows the PSG 4 ganglioside fraction following treatment by protease type VI (lanes 2 and 6), protease type $\mathrm{V}$ (lanes 3 and 7 ) and trypsin (lanes 4 and 8). Comparison with control PSG 4 (lanes 1 and 5) shows that there is no significant change of immune reactivity following each of these treatments. Furthermore we have applied varying amounts of the PSG 4 gangliosides to polyacrylamide gels and stained them with the highly sensitive silver staining procedure. No bands were detected (results not shown). These results argue strongly against the Chol-1 antigens' being peptidic contaminants of the ganglioside preparations.

Non-gangliosidic acidic glycolipids are also known to contaminate ganglioside preparations. Therefore in order to demonstrate the presence of sialic acid in the Chol- 1 antigens and hence to confirm their gangliosidic nature we have carried out neuraminidase treatment of the PSG 4 ganglioside fraction. When the PSG 4 ganglioside fraction was digested with neuraminidase in conditions such that all the major gangliosides are metabolized to GM1, immunoreactivity against the Chol-1 antigens is lost (Fig. 3). In conditions where the digestion is less complete immunoreactive bands migrating with higher RF's can be seen $^{8}$. These results demonstrate the presence of sialic acid in the Chol-1 antigens. Together with the evidence presented earlier ${ }^{12}$ these data provide evidence in favour of the gangliosidic nature of Chol-1.
In other work (Giuliani et al., submitted for publication) we have purified Chol- $1 \beta 4000$ times and all the properties of the purified antigen are in agreement with its being gangliosidic in nature.

\section{Developmental expression of Chol-1 in rat brain}

Gangliosides extracted from the brains of both embryonic and postnatal animals containing between 3 and $5 \mu \mathrm{g}$
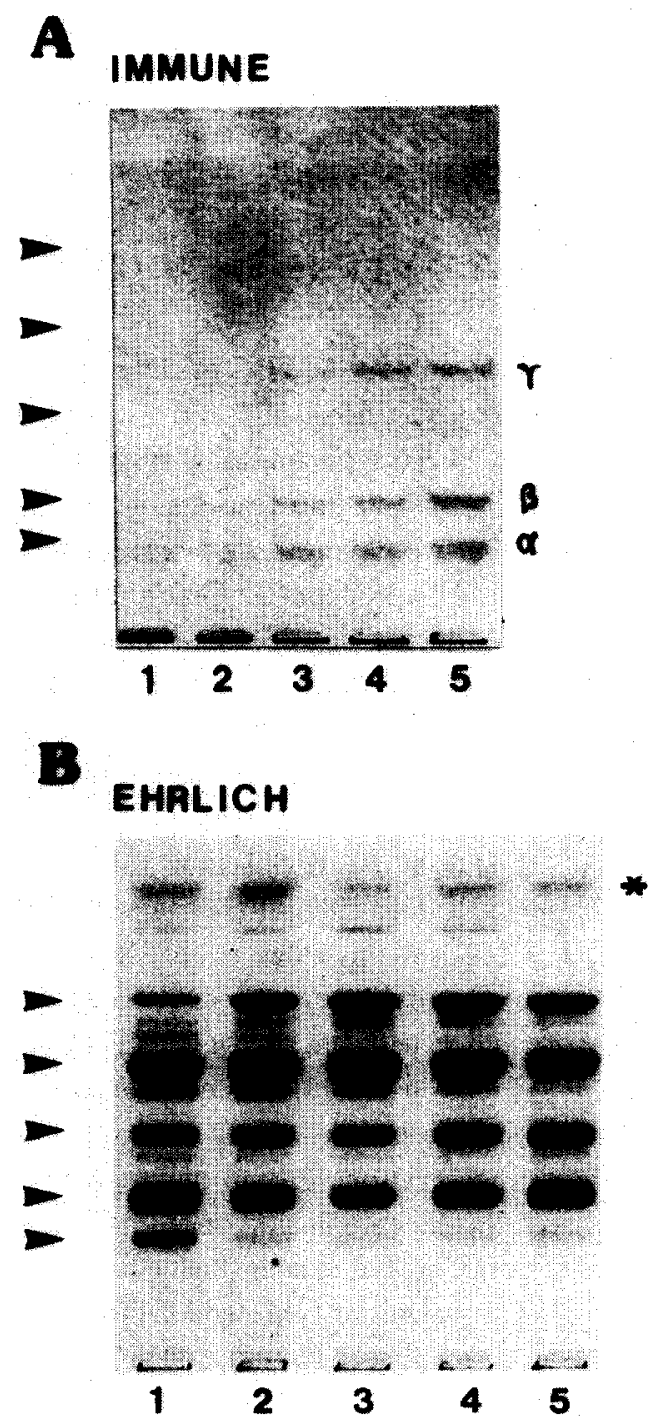

Fig. 6. The developmental expression of Chol-1 in rat hippocampus. A: gangliosides prepared from 5, 10, 20 and 30 day and adult hippocampus containing 5, 5, 4, 3 and $3 \mu$ g sialic acid, respectively were separated on TLC and stained with anti-Chol-1 antiserum (lanes 1-5). The expression of the immunoreactive Chol-1 gangliosides can be seen to increase relative to the total gangliosides during development, Chol- $1 \alpha$ and $\beta$ first being detected 10 days postnatally and Chol-1 $\gamma 20$ days postnatally. Different amounts of gangliosides were loaded in each lane so that the intensity of the immune staining would be within the limits of the linearity of the system (see also legend to Fig. 4). B: gangliosides prepared from hippocampi of the same developmental series were separated on TLC ( $3 \mu \mathrm{g}$ sialic acid per lane) and stained with Ehrlich's reagent (lane 1-5, respectively). For asterisk and arrowheads see legend to Fig. 4. 

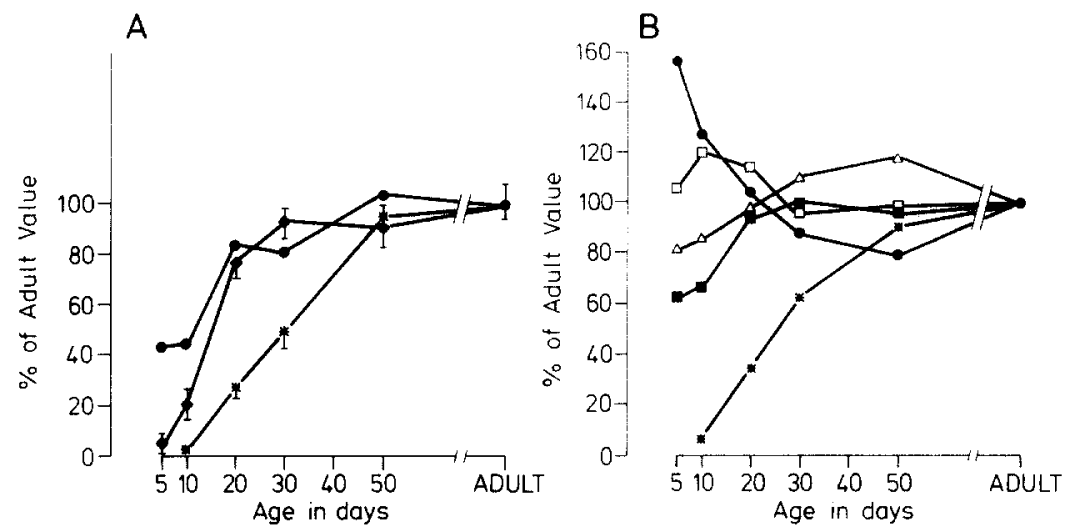

Fig. 7. A: the quantitative developmental expression of ChAT, Chol-1 and ganglioside-bound sialic acid in rat hippocampus. The expression of Chol- 1 can be seen to increase in parallel with that of ChAT though it appears to lag behind the expression of ChAT by about 20 days. Data were calculated per unit of wet weight and expressed as percentages of adult values. Circles, ganglioside-bound sialic acid; asterisks, Chol-1; diamonds, ChAT. For Chol-1 and ChAT, each point represents the mean \pm S.D. of 3 experiments; for sialic acid, the results of a typical experiment are shown. B: developmental expression of Chol-1 and major gangliosides. Data were calculated and expressed as indicated in the legend of Fig. 5B. Asterisks, Chol-1; empty triangles, GM1; empty squares, GDla; filled squares, GD1b; filled circles, GT1b.

sialic acid were immune stained on TLC as described in the 'Materials and Methods'. Chol- $1 \alpha, \beta$ and $\gamma$ were first detected at 5,10 and 20 days postnatally, respectively (Fig. 4). Since ChAT is the enzyme responsible for the synthesis of acetylcholine we have used its expression as an index of cholinergic synaptogenesis. Quantitation of both ChAT and Chol-1 during development shows that the level of Chol- 1 closely follows that of ChAT (Fig. 5A). Though the major gangliosides are also increasing in concentration during this period ${ }^{28,42}$ as shown by the increase in ganglioside-bound sialic acid per unit of weight (Fig. 5A), it should be noted that they have already reached approximately $50 \%$ of their adult levels by postnatal day 5 . Chol-1 at this stage is only $8 \%$ of its adult level and it rises rapidly during the next 50 days in a manner closely correlated with the rise of ChAT. In Fig. 5B, the pattern of expression of Chol- 1 is compared with that of the major brain gangliosides. Data are expressed here as the relative concentration per unit of gangliosidebound sialic acid. Within the ganglioside pool, the rate of increase during this period in the concentration of Chol-1 is considerably higher than that of GM1 and GD1b while the relative concentrations of GT1b and GD1a fall.

The close correlation between the expression of Chol-1 and ChAT combined with its general period of expression in the brain suggests that Chol-1 may be involved in processes related to the formation and/or maturation of cholinergic nerve terminals. In order to establish whether or not Chol-1 was expressed by the presynaptic plasma membrane before synaptogenesis, gangliosides were extracted from growth cone particles isolated from 5-day postnatal rat brain. Five micrograms of gangliosidebound sialic acid prepared from growth cones and 5 -day-old brain were loaded on TLC and immune stained with anti-Chol-1 antiserum. Chol-1 was not detected in growth cones (result not shown); however, since it is detected in the whole brain at this time in development, Chol -1 is probably located in the more mature cholinergic synapses.

In order to establish with greater resolution at which stage Chol-1 is expressed during synaptogenesis we have studied its expression in two systems in which the major events of cholinergic synaptogenesis have been thoroughly described.

The developmental expression of Chol-1 in the hippocampus of rat

Though the cell bodies of cholinergic interneurons have been found in the hippocampus ${ }^{23}$ lesions of the fimbria demonstrate that the vast majority of the cholinergic innervation of the hippocampus originates from cell bodies in the basal forebrain'. Since such lesions

Fig. 8. Developmental expression of Chol-1 in the rat neuromuscular junction. Sections taken from 3- (A, B), 10-day-old (C, D) and adult (E-H) rat intercostal muscle were double-stained with rhodamine-labelled $\alpha$-bungarotoxin (A, C, E and $G$ ) and with affinity-purified anti-Chol-1 antiserum $(B, D$ and $F$ ) or sheep non-immune serum $(H)$. The bound antibodies were visualized using a fluorescein-conjugated second antibody. Chol-1 immunoreactivity is first detected at postnatal day 3 . The staining intensity at this stage is low but clearly distinguishable from that of the non-immune serum. The intensity of the staining increases during development as shown by the 10-day and adult sections. The co-localization between $\alpha$-bungarotoxin (which binds specifically to the nicotinic acetylcholine receptor) and the antigen(s) recognized by anti-Chol-1 antiserum confirms the localization of the Chol-1 immunoreactivity at the level of the neuromuscular junction. Only very faint, non-specific staining, mainly distributed on the connective tissue that surrounds the muscle bundles, was observed using control sheep serum (H). Bars $=10 \mu \mathrm{m}$. 

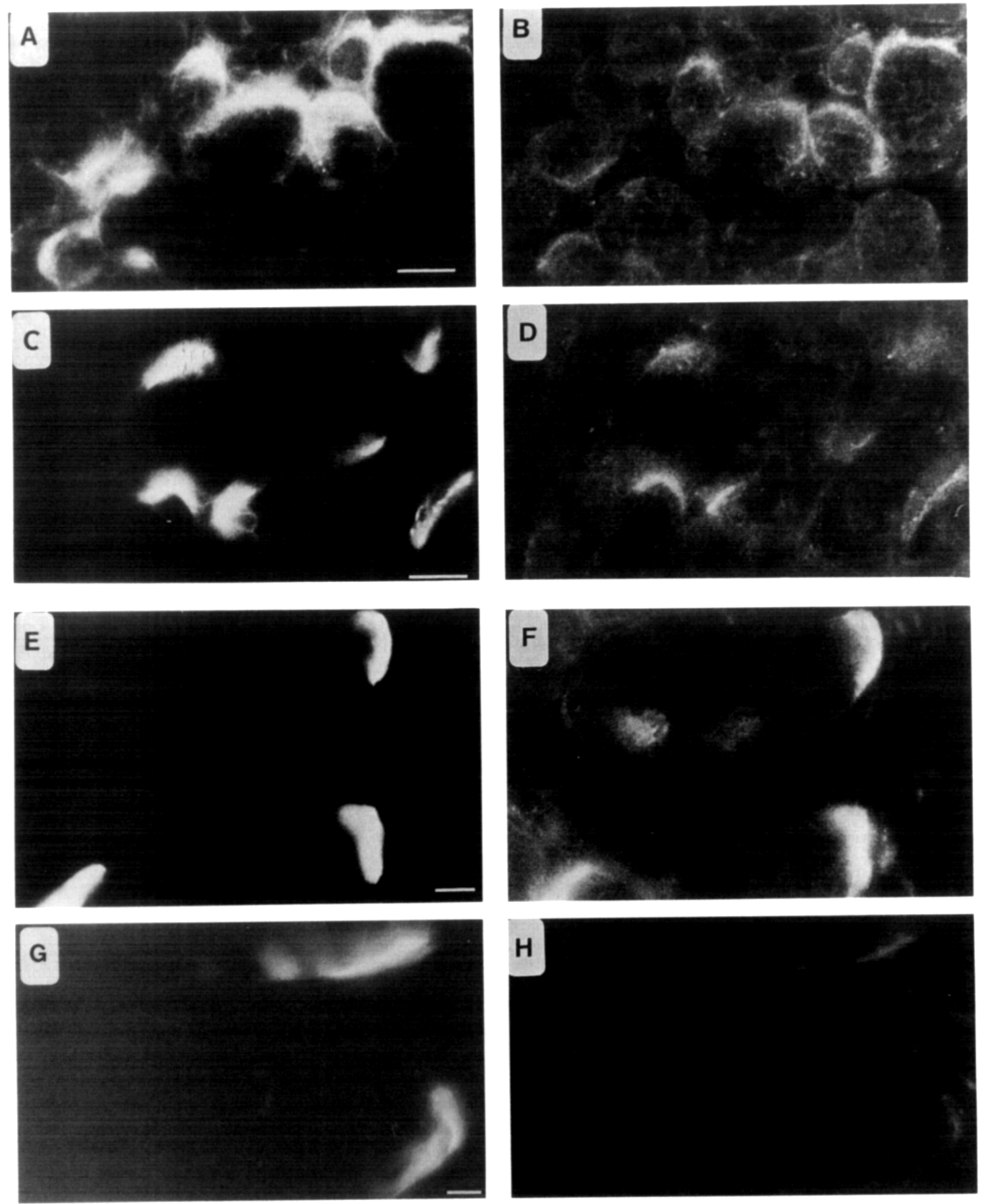
result in an approximately $90 \%$ loss of ChAT in the ipsilateral hippocampus one may conclude that no more than $10 \%$ of the cholinergic innervation of the hippocampus is due to endogenous interneurons. Thus the change in the level of cholinergic markers, including Chol-1, in the hippocampus during development will primarily reflect cholinergic synaptogenesis rather than other processes.

Gangliosides from the hippocampi of rats, ages ranging from 5 to 50 days postnatal, and from adult rat hippocampus were separated on TLC and immune stained. Chol- $1 \alpha$ and $\beta$ were first detected at postnatal day 10 and Chol- $1 \gamma$ at day 20 (Fig. 6). The quantitation of ChAT and Chol-1 during development (Fig. 7A) shows that there is a close correlation between these two markers. The expression of Chol-1 lags behind that of ChAT suggesting that it is expressed by the cholinergic terminals later. The comparison of the pattern of expression of Chol-1 with that of the major gangliosides confirms the results obtained in total brain. Chol-1 rises rapidly to almost the adult level during the first 50 days of life. In the same period the relative concentrations of GM1 and GD1b show smaller increases while those of GD1a and GT1b fall (Fig. 7B).

\section{Developmental expression of Chol-1 at the neuromuscular junction of the intercostal muscle}

Chol-1 can first be detected at the neuromuscular junction colocalizing with $\alpha$-bungarotoxin (a highly specific label for the acetylcholine receptor) at postnatal day 3 (Fig. 8). Though the staining intensity at this stage is low it can be seen to increase during development as shown by the 10 day and adult sections.

\section{DISCUSSION}

Previous work done in this laboratory has shown that the anti-Chol-1 antiserum recognizes minor gangliosides located selectively on the cell surface of cholinergic neurons. The expression of these gangliosides appears to be developmentally regulated. They are first detected in rat brain, respectively at 5,10 and 20 days postnatally. Their level of expression closely follows that of the cholinergic marker ChAT. Because Chol-1 first appears in the period during which synaptogenesis is taking place, as measured by the expression of several nerve terminal related markers e.g. synaptophysin ${ }^{24}$, acetylcholinesterase $(\mathrm{AChE})^{13}$ and ChAT (this paper, Fig. 5), we have studied the expression of Chol-1 in two systems within which the development of cholinergic synapses has been well characterized: the neuromuscular junction of the intercostal muscle and the hippocampus of rat.

Complete section of the fornix fimbria interrupts the septohippocampal fibre tract and causes the degeneration of about $90 \%$ of the cholinergic system of the hippocampus, as measured by the fall in the level of $\mathrm{ChAT}^{10}$. It has also been established that concomitant falls in the level of Chol- 1 are caused by such lesions ${ }^{8}$. Thus changes in the levels of cholinergic markers in the hippocampus during development will primarily reflect cholinergic synaptogenesis rather than any other process occurring in the cholinergic neurons at this time. Orthograde and retrograde labelling experiments have shown that fibres from the cholinergic cell bodies of the medial septum first begin innervating the hippocampus as early as foetal day $16^{29}$. Though the distribution of terminals is at first diffuse, between postnatal days 3 and 14 hippocampal regions which are most innervated in the adult animals become progressively more innervated and terminals in areas which do not exhibit cholinergic innervation in adults are progressively eliminated ${ }^{29}$. It is interesting to note, however, that the presence of cholinergic markers such as ChAT, AChE and high affinity choline uptake do not appear until postnatal days 1,4 and 6 after the cholinergic fibres enter the hippocampus respectively ${ }^{29}$. Chol-1 is first detected 10 days postnatal. It increases rapidly in concentration during the next 30 days paralleling the increase shown by ChAT though lagging behind by about 20 days. This strongly suggests that the expression of Chol- 1 is a membrane modification very late in the development of the cholinergic synapse. This is consistent with the finding that Chol-1 is not found in the growth cone particles (immature nerve terminals) in 5 -day old brain but is found in the whole brain at this time, suggesting that Chol- 1 is present only in the more mature cholinergic elements at this time.

Further support for this conclusion came from the finding that Chol-1 is first detected in the neuromuscular junction three days postnatally. At this time the synapses in the intercostal muscle have already formed and are electrophysiologically functional ${ }^{7}$. Thus the expression of Chol-1 is an occurrence late in the maturation of the cholinergic synapse both in the central and the peripheral nervous system of the rat. Similar results were obtained in a study of the developmental expression of Chol- 1 in the electric organ of Torpedo marmorata ${ }^{14}$

Gangliosides have been implicated in a great variety of processes related to the development of the nervous system e.g. as stimulators of neurite outgrowth ${ }^{5,11}$, as recognition molecules and adhesion molecules ${ }^{6,27}$, as modulators of cell growth ${ }^{41}$ and in cell motility ${ }^{39}$. The late onset of expression of Chol-1 would seem to rule out the possibility that it is involved in cell motility or that it functions as a recognition molecule during synaptogenesis.

However, there is increasing evidence that gangliosides 
are effective in modulating receptors for various trophic factors and proteins. Anti-ganglioside antibodies inhibit nerve growth factor induced sprouting from chick dorsal root ganglia ${ }^{40}$. Gangliosides appear to modulate the receptor for platelet derived growth factor ${ }^{3}$, epidermal growth factor ${ }^{19}$ and ganglioside GD2 has been shown to modulate the affinity of a melanoma receptor for the extracellular matrix protein vitronectin by associating with the receptor in a calcium dependent manner ${ }^{6}$.

Interestingly, Chol-1 is first being expressed in the hippocampus during the period in which the action of nerve growth factor on septal neurons innervating the hippocampus changes from inducing the production of ChAT to promotion of cell survival ${ }^{20}$. In the neuromuscular junction Chol-1 is first detected during the period when the multiple innervation of muscle fibres is being

\section{REFERENCES}

1 Atack, J.R., Perry, E.K., Bonham, J.R., Boakes, R. and Candy, J.M., Loss and recovery of acetylcholinesterase molecular forms in the fornix-lesioned rat hippocampus, Neurosci. Lett., 79 (1987) 174-184.

2 Borroni, E., Ferretti, P., Obrocki, J. and Whittaker, V.P., Characterization and distribution of Chol-1, a new cholinergicspecific antigen. In S. Tuček (Ed.), Synaptic Transmitters and Receptors, Academia, Prague, in co-publication with J. Wiley and Sons, Chichester, 1987, pp. 55-60.

3 Bremer, E.G., Hakomori, S., Bowen-Pope, D.F., Raines, E. and Ross, R., Ganglioside-mediated modulation of cell growth, growth factor binding and receptor phosphorylation, J. Biol. Chem., 259 (1984) 6818-6825.

4 Brockaus, J., Magnani, J.L., Blaszczyk, S.Z., Koprowski, H., Karlsson, K., Larson, G. and Ginsburg, V., Monoclonal antibodies directed against the human $\mathrm{Le}^{\mathrm{b}}$ blood group antigen, J. Biol. Chem., 256 (1981) 13223-13225.

5 Cannella, M., Roisen, F., Ogawa, T., Sugimoto, M. and Ledeen, R., Comparison of eip- $\mathrm{GM}_{3}$ with $\mathrm{GM}_{3}$ and $\mathrm{GM}_{1}$ as stimulators of neurite outgrowth, Dev. Brain Res., 39 (1988) 137-143.

6 Cheresh, D.A., Pytela, R., Pierschbacher, M.D., Klier, F.G., Ruoslahti, E. and Reisfeld, R.A., An Arg-Gly-Asp-directed receptor on the surface of human melanoma cells exists in a divalent cation-dependent functional complex with the disialoganglioside GD2, J. Cell Biol., 105 (1987) 1163-1173.

7 Dennis, M.J., Ziskind-Conheim, L. and Morris, A.J., Development of neuromuscular junctions in rat embryos, Dev. Biol., 81 (1981) 266-279.

8 Derrington, E., Masco, D. and Whittaker, V.P., Confirmation of the cholinergic specificity of the Chol-1 gangliosides in mammalian brain using affinity-purified antisera and lesions affecting the cholinergic input to the hippocampus of rat, $J$. Neurochem., (1989) (in press).

9 Dodd, J. and Jessel, T., Lactoseries carbohydrates specify subsets of dorsal root ganglion neurons projecting to the superficial dorsal horn of rat spinal cord, J. Neurosci., 5 (1985) 3278-3294.

10 Dravid, A.R. and Van Deuson, E.B., Recovery of choline acetyltransferase and acetylcholinesterase activities in the ipsilateral hippocampus following unilateral partial transection of the fimbria, Brain Res., 277 (1983) 169-174.

11 Ferrari, G., Fabris, M. and Gorio, A., Gangliosides enhance neurite outgrowth in PC12 cells, Dev. Brain Res., 8 (1983) 215-221.

12 Ferretti, P. and Borroni, E., Putative cholinergic-specific gan- eliminated ${ }^{7}$. We are currently trying to establish whether this correlation between the expression of the Chol-1 gangliosides and the establishment of the adult pattern of the cholinergic innervation of the hippocampus and the neuromuscular junction is not merely coincidental. Thus we are investigating whether Chol-1 might be expressed either to modulate the nerve growth factor receptor in the hippocampus or a putative cell adhesion complex which is acting to stabilize cholinergic synapses.

Acknowledgements. The authors thank Dr G.I. Kristjansson for isolating growth cones and Professor V.P. Whittaker for critically reading the manuscript. E.D. was supported by fellowships from the Max-Planck-Gesellschaft and Fidia Pharmaforschung GmbH, and E.B. by the Sonderforschungsbereich 236 and Grant Wh $1 / 5-1$ of the Deutsche Forschungsgemeinschaft to Prof. Whittaker. To these sponsors we express our sincere gratitude.

gliosides in guinea-pig forebrain, $J$. Neurochem., 46 (1986) 1888-1894.

13 Fiedler, E.P., Marks, M.J. and Collins, A.C., Development of cholinergic enzymes and receptors in mouse brain, J. Neuro. chem., 49 (1987) 983-990.

14 Fiedler, W., Borroni, E. and Ferretti, P., An immunohistochemical study of synaptogenesis in the electric organ of Torpedo marmorata by use of antisera to vesicular and presynaptic plasma membrane components, Cell Tiss. Res., 246 (1986) 439-446.

15 Fonnum, F., A rapid radiochemical method for the determination of choline acetyltransferase, $J$. Neurochem., 24 (1975) 407-409.

16 Gordon-Weeks, P.R. and Lockerbie, R.O., Isolation and partial characterization of neuronal growth cones from neonatal rat forebrain, Neuroscience, 13 (1984) 119-136.

17 Grunwald, G.B., Fredman, P., Magnani, J.L., Trisler, D., Ginsburg, V. and Nirenberg, M., Monoclonal antibody 18B8 detects gangliosides associated with neuronal differentiation and synapse formation, Proc. Natl. Acad. Sci. U.S.A., 82 (1985) 4008-4012

18 Hakomori, S., Glycosphingolipids in cellular interaction, differentiation and oncogenesis, Annu. Rev. Biochem., 50 (1981) 733-764.

19 Hanai, N., Dohi, T., Nores, G. and Hakomori, S., A novel ganglioside, De-N-acetyl-GM (II $^{3} \mathrm{NeuNH}_{2} \mathrm{LacCer}$ ), acting as a strong promotor for epidermal growth factor receptor kinase and as a stimulator for cell growth, J. Biol. Chem., 263 (1988) 6296-6301.

20 Hatanaka, H., Tsukui, H. and Nihonmatsu, I., Developmental change in the nerve growth factor action from induction of choline acetyltransferase to promotion of cell survival in cultured basal forebrain cholinergic neurons from postnatal rats, Dev. Brain Res., 39 (1988) 85-95.

21 Jessen, K.R., Morgan, L., Brammer, M. and Mirsky, R., Galactocerebroside is expressed by non-myelin-forming Schwann cells in situ, J. Cell Biol., 101 (1985) 1135-1143.

22 Jones, R.T., Walker, J.H., Richardson, P.J., Fox, G.Q. and Whittaker, V.P., Immunohistochemical localization of cholinergic nerve terminals, Cell Tiss. Res., 218 (1981) 355-373.

23 Kimurah, H., McGeer, P., Peng, J. and McGeer, E., The central cholinergic system studied by choline acetyltransferase immunohistochemistry in the cat, J. Comp. Neurol., 200 (1981) 151-201.

24 Knaus, P., Betz, H. and Rehm, H., Expression of synaptophysin during postnatal development of the mouse brain, J. Neurochem., 47 (1986) 1302-1304.

25 Ledeen, R.W., Gangliosides. In A. Lajtha (Ed.), Handbook of 
Neurochemistry, Plenum Press, New York/London, 1983, pp. 41-90.

26 Lowry, O.H., Rosenbrough, N.J., Farr, A.L. and Randall, R.J., Protein measurement with the Folin phenol reagent, J. Biol Chem., 193 (1951) 265-275.

27 Marchase, R.B., Biochemical investigation of retinotectal adhesive specificity, J. Cell Biol., 75 (1977) 237-257.

28 Merat, M. and Dickerson, J., The effect of development on the gangliosides of rat and pig brain, J. Neurochem., 20 (1973) 873-880.

29 Milner, T., Loy, R. and Amaral, D., An anatomical study of the development of the septo-hippocampal projection in the rat. Dev. Brain Res., 8 (1983) 343-371.

30 Obata, K. and Oide, M., Effects of glycolipids on in vitro development of neuromuscular junction, Nature (Lond.), 266 (1977) 369-371

31 Obrocki, J. and Borroni, E., Immunocytochemical evaluation of a cholinergic-specific ganglioside antigen (Chol-1) in the centra nervous system of the rat, Exp. Brain Res., 72 (1988) 71-82.

32 Partridge, S.M., Filter-paper partition chromatography of sugars, Biochem. J., 42 (1948) 238-248.

33 Raff, M.C., Fields, K.L., Hakomori, S.I., Mirsky, R. and Pruss, R.M., Cell-type specific markers for distinguishing and studying neurons and the major classes of glial cells in culture, Brain Res., 174 (1978) 283-308.

34 Ravdin, P. and Axelrod, D., Fluorescent tetramethylrhodamine derivates of $\alpha$-bungarotoxin: preparation, separation and characterization, Anal. Biochem., 80 (1977) 585-592.

35 Richardson, P.J., Presynaptic distribution of the cholinergicspecific antigen Chol-1 and $5^{\prime}$-nucleotidase in rat brain, as determined by complement-mediated lysis of neurotransmitters, J. Neurochem., 41 (1983) 640-648.

36 Richardson, P.J., Siddle, K. and Luzio, J.P., Immunoaffinity purification of intact, metabolically active, cholinergic nerve terminals from mammalian brain, Biochem. J., 219 (1984) 647-654.

37 Richardson, P.J., Walker, J.H., Jones, R.T. and Whittaker, V.P., Identification of a cholinergic-specific antigen Chol-1 as a ganglioside, J. Neurochem., 38 (1982) 1605-1614

38 Roisen, F.J., Bartfeld, M., Magelle, R. and Yorke. G. Ganglioside stimulation of axonal sprouting in vitro, Science, 214 (1981) $577-578$.

39 Schlosshauer, B., Blum, A., Mendez-Otero, R., Barnstable. C. and Constantine-Paton, M., Developmental regulation of ganglioside antigens recognized by the JONES antibody, $J$. Neurosci., 8 (1988) 580-592.

40 Schwartz, M. and Spirman, N., Sprouting from chicken embryo dorsal root ganglia induced by nerve growth factor is specifically inhibited by affinity-purified antiganglioside antibodies, Proc. Natl Acad. Sci. U.S.A., 79 (1982) 6080-6083

41 Spiegel, S. and Fishman, P., Gangliosides as bimodal regulators of cell growth, Proc. Natl. Acad. Sci. U.S.A., 84 (1987) 141-145.

42 Suzuki, K., The pattern of mammalian brain gangliosides - III. Regional and developmental differences, $J$. Neurochem. 12 (1965) 969-979.

43 Svennerholm, L., Quantitative estimation of sialic acids. II. A colorimetric resorcinol-hydrochloric acid method, Biochim. Biophys. Acta, 24 (1957) 604-611.

44 Svennerholm, L., Ganglioside designation, Adv. Exp. Med. Biol., 125 (1980) 11

45 Svennerholm, L., Ganglioside and synaptic transmission. In L. Svennerholm, P. Mandel, H. Dreyfus and P.F. Urban (Eds.) Advance in Experimental Medicine and Biology, Vol. 125, Plenum Press, New York, London, 1980, pp. 533-542.

46 Tettamanti, G., Bonali, F., Marchesini, S. and Zambotti, V., A new procedure for the extraction, purification and fractionation of brain gangliosides, Biochim. Biophys. Acta, 296 (1973) $160-170$

47 Whittaker, V.P. and Borroni, E., Cholinergic-specific antigens In V.P. Whittaker (Ed.), The Cholinergic Synapse, Handbook of Experimental Pharmacology, Vol. 86, Springer, Heidelberg, 1988, pp. 447-463.

48 Young, W.W., Laine, R.A. and Hakomori, S.I., Covalent attachment of glycolipids to solid supports and macromolecules. In V. Ginsburg (Ed.), Methods in Enzymology, Vol. 50, Academic Press, New York, 1978, pp. 137-140. 